\title{
Vorhersage des Phasenrauschens in optischen Messsystemen mit strukturierter Beleuchtung
}

Marc Fischer, Marcus Petz, Rainer Tutsch

Institut für Produktionsmesstechnik, Technische Universität Braunschweig

Schleinitzstraße 20, 38106 Braunschweig

Tel.: 0531-391-7023, Fax: 0531-391-5837, E-Mail: marc.fischer@tu-braunschweig.de

\section{Kurzfassung}

Optische Messsysteme mit strukturierter Beleuchtung basieren häufig auf einer Ortscodierung durch phasenverschobene sinusförmige Intensitätsmuster. Durch Auswertung der beobachteten Sequenz von Intensitätswerten kann für jedes Pixel der Kamera unabhängig von seiner Nachbarschaft ein Phasenwert berechnet werden. Die zufälligen Abweichungen bei dieser Phasenbestimmung werden im Wesentlichen von den Rauscheigenschaften der verwendeten Kamera bestimmt. Da bei Messungen mit strukturierter Beleuchtung im industriellen Umfeld im Allgemeinen auf Wiederholungsmessungen verzichtet wird, wäre es wünschenswert, im Sinne eines vollständigen Messergebnisses, neben dem Messwert auch eine Schätzung für die Unsicherheit zu erhalten. Diese kann dann sowohl für eine allgemeine Bewertung der Messung aber insbesondere auch im Rahmen der Berechnung von 3D-Punkten und dem Anpassen von geometrischen Standard-Elementen als Maß für die Zuverlässigkeit der Einzelmesswerte eingesetzt werden. Da bisherige Untersuchungen zu diesem Thema entweder auf sehr einfachen Rauschmodellen basieren oder die beschreibenden Parameter nicht einfach zugänglich sind, wird in dieser Arbeit eine Methode vorgestellt, mit der sich ein Schätzwert für das Phasenrauschen direkt aus den aufgezeichneten Grauwerten berechnen lässt. Das Modell basiert dabei auf einer Norm zur Charakterisierung von Bildsensoren (EMVA 1288), so dass die entsprechenden Parameter direkt einem konformen Datenblatt entnommen werden können. Alternativ wird in dieser Arbeit auch eine einfache Methode beschrieben, mit der die Parameter ohne zusätzlichen Geräteaufwand experimentell ermittelt werden können.

\section{Einleitung}

Optische Messsysteme mit strukturierter Beleuchtung basieren auf einer Ortscodierung des Messobjekts bzw. einer Referenzfläche durch ortsabhängige Helligkeitsverteilungen. Bei matten Oberflächen kommen dabei Streifenprojektions- und bei spiegelnden Oberflächen Streifenreflexionsverfahren zum Einsatz. Als Muster wird dabei häufig eine Sequenz von phasenverschobenen Sinusmustern eingesetzt, da diese neben einer hohen Ortsauflösung auch eine pixelweise unabhängige Auswertung ermöglichen. Das Grundprinzip der Auswertung ist in Abbildung 1 für den weit verbreiteten 4-Schritt-Algorithmus (vier um je $90^{\circ}$ verschobene Sinusmuster) dargestellt.
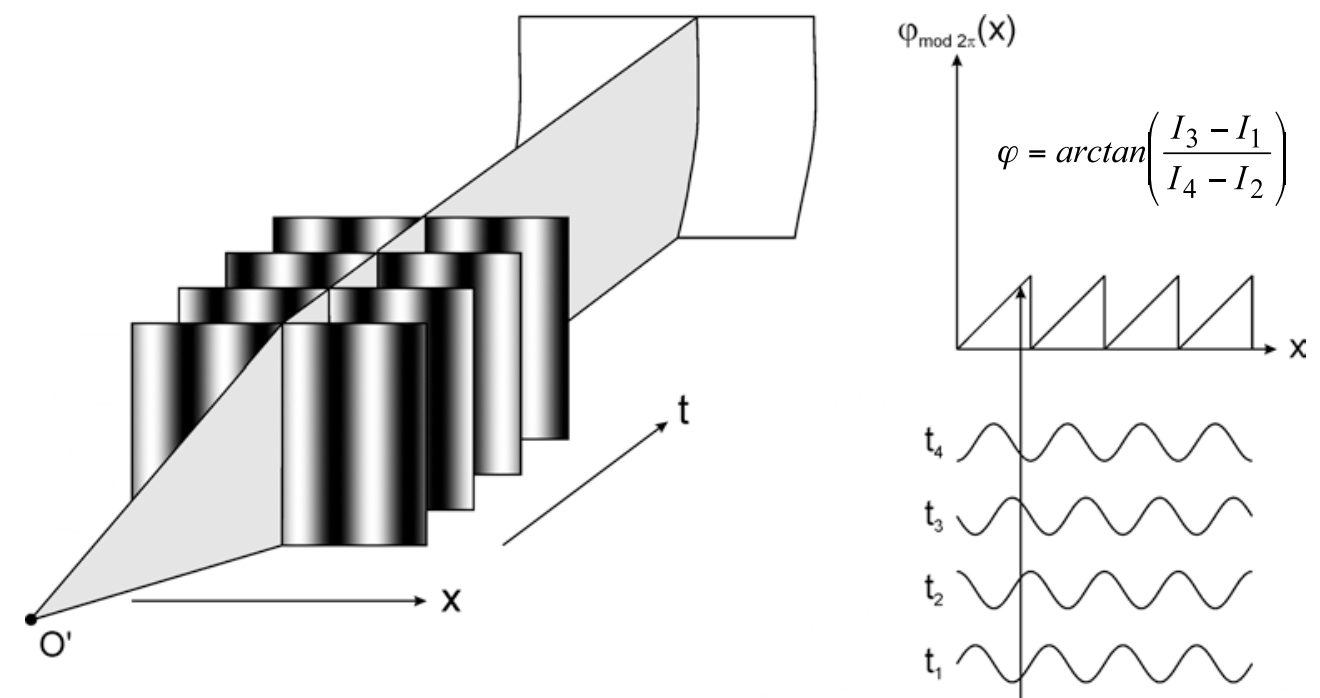

Abbildung 1: Grundprinzip der Ortscodierung durch phasenverschobene Sinusmuster 
Aus den vier sequentiell zu den Zeitpunkten $t_{1}$ bis $t_{4}$ aufgezeichneten Intensitäten kann senkrecht zur Streifenrichtung in jedem Pixel der Kamera eine Ortsinformation $x$, zunächst $2 \pi$-periodisch, codiert werden. Die Erweiterung auf eine im gesamten Messbereich eindeutige Ortscodierung erfolgt durch Anwendung von Entfaltungsalgorithmen, welche z.B. auf der zusätzlichen Anzeige von Graycode-Sequenzen oder auf der Auswertung verschiedener Wellenlängen basieren. Die Bestimmung von 3D-Punkten erfolgt dann durch Triangulation, wobei die Messdaten anschließend im Allgemeinen noch weiterverarbeitet und beispielsweise geometrische Funktionselemente in die Punktewolke eingepasst werden.

Die in optischen Messsystemen mit strukturierter Beleuchtung eingesetzten Algorithmen zur Phasenbestimmung sind aus der Phasenschiebe-Interferometrie übernommen worden. Auf diesem Gebiet wurden in den letzten Jahrzehnten leistungsfähige Algorithmen entwickelt, welche den Einfluss systematischer Effekte in der Interferometrie, wie z.B. Phasenschiebefehler, Kennlinienfehler und Vibrationen minimieren [1]. Aufgrund der hohen Empfindlichkeit interferometrischer Verfahren sind diese Effekte gegenüber stochastischen Rauscheinflüssen dominierend. Dem Thema Phasenrauschen wurde daher in der Forschung deutlich weniger Aufmerksamkeit zuteil und die meisten Arbeiten basieren auf vereinfachten Annahmen zum Rauschverhalten des eingesetzten Bildsensors [2][3][4].

Die in dieser Arbeit betrachteten Messverfahren hingegen arbeiten mit inkohärentem Licht und basieren auf einer Winkelmessung. Sie sind daher inhärent deutlich robuster gegenüber Vibrationen und Schwankungen der Umgebungsbedingungen. Häufig werden zur Verkörperung der Muster digitale Displays (Projektor / Monitor) eingesetzt. Diese ermöglichen die Erzeugung der Phasenverschiebungen mit einer sehr hohen Präzision, die im Wesentlichen nur durch die photolithographische Fertigungsgenauigkeit begrenzt ist. Der aufgrund der digitalen Projektion entstehende systematische Aliasing-Fehler kann durch eine Defokussierung des Musters deutlich reduziert werden [5]. Zusammengenommen führen die beschriebenen Effekte zu einer deutlich höheren Relevanz stochastischer Einflüsse für Messverfahren mit strukturierter Beleuchtung. Die stochastischen Abweichungen der gemessenen Phaseninformation resultieren dabei im Wesentlichen aus dem Rauschen der in der Kamera aufgezeichneten Intensitäten.

Für den Anwender besteht dabei das Problem, dass er eine Aussage über die Unsicherheit der Phasenbestimmung nur durch Wiederholungsmessungen erhalten würde. Auf diese wird jedoch bei industriellem Einsatz der Messverfahren aus Kostengründen im Allgemeinen verzichtet. Stattdessen erfolgt eine allgemeine Spezifikation der Messunsicherheit durch Messung an kalibrierten Probekörpern. Insbesondere im Hinblick auf die Weiterverarbeitung der Daten durch Anpassung von geometrischen Standardelementen oder der Fusion der Daten unterschiedlicher Messungen führt der Mangel an Informationen über die Zuverlässigkeit der Phaseninformation von Einzelmesswerten zu statistisch suboptimalen Ergebnissen aufgrund fehlender Gewichtungsmöglichkeit. Eine mögliche Lösung dieses Problems stellt die Schätzung des Phasenrauschens der Einzelmesswerte auf der Grundlage eines Kamerarauschmodells dar, wobei ein entscheidendes Kriterium für den Anwender neben der Modellgüte insbesondere die einfache Verfügbarkeit der Modellparameter darstellt.

In einer Norm der European Machine Vision Association mit dem Titel „EMVA Standard 1288 - Standard for Characterization of Image Sensors and Cameras“ (EMVA 1288) [6] wird ein Kameramodell zur allgemeinen Charakterisierung von Bildsensoren und Kameras beschrieben. Der aktuelle Normentwurf wurde im November 2010 veröffentlicht und ermöglicht eine objektive und vergleichbare Spezifikation. Das Kameramodell berücksichtigt neben normalverteiltem Dunkelrauschen insbesondere auch Poisson-verteiltes Photonenrauschen und die Quantisierung der Grauwerte. Es weist daher eine hohe Modellgüte auf und wurde bereits erfolgreich für die Analyse von optischen Messsystemen eingesetzt [7][8]. Es existiert bislang jedoch keine zufriedenstellende Methode, welche die Erkenntnisse zum Thema Phasenrauschen mit dem leistungsfähigen Kameramodell der EMVA 1288 allgemeingültig zusammenführt und eine Schätzung der stochastischen Abweichungen direkt aus den aufgezeichneten Intensitäten ermöglicht.

Nach einer allgemeinen Erläuterung der statistischen Eigenschaften von Phasenschiebe-Algorithmen wird daher im Rahmen dieser Arbeit für die Klasse der symmetrischen M-Schritt Algorithmen ein auf dem Kameramodell der EMVA 1288 basierendes Phasenrauschmodell hergeleitet und durch Simulationen und Experimente validiert. Da das Kameramodell aus der EMVA 1288 übernommen wurde, können die Modellparameter vom Anwender direkt einem konformen Datenblatt entnommen werden. Für den Fall, dass die Parameter nicht zur Verfügung stehen, wird in dieser Arbeit ebenfalls eine einfache Methode zur experimentellen Ermittlung beschrieben, welche sich ohne zusätzlichen Geräteaufwand direkt mit dem zu charakterisierenden Messsystem umsetzen lässt. Auf der Grundlage des Phasenrauschmodells erfolgt dann die Formulierung einer Auswertemethode, welche es ermöglicht, zu jedem Messwert auch eine Schätzung für die Unsicherheit der Phasenbestimmung anzugeben. Abschließend erfolgt eine experimentelle Analyse der Genauigkeit der Modellvorhersage. 


\section{Statistische Eigenschaften von Phasenschiebe-Algorithmen}

Für die im Rahmen dieser Arbeit betrachteten optischen Messsysteme mit strukturierter Beleuchtung auf der Grundlage von phasenverschobenen sinusförmigen Streifenmustern erfolgt die Aufnahme der unterschiedlichen Phasenverschiebungen sequentiell. Die in einem Pixel der Kamera im Bild $i$ der Sequenz beobachtete Intensität $I_{i}$ kann dabei als Summe eines konstanten und eines modulierten Signalanteils beschrieben werden:

$$
I_{i}=I^{\prime}+I^{\prime \prime} \cos \left(\varphi+\psi_{i}\right) ; \quad i=1, \ldots, M
$$

Hierbei bezeichnet $\varphi$ den zu berechnenden Phasenwinkel, $\psi_{i}$ die Phasenverschiebung des entsprechenden Musters und $M$ die Gesamtanzahl der verwendeten Muster. Für eine allgemeingültige Diskussion ist es sinnvoll, den absoluten Bezug der Intensitätsgrößen $I^{\prime}$ und $I^{\prime \prime}$ zu eliminieren. Durch die Definition von

$$
I^{\prime}=\beta I_{\text {sat }}
$$

und

$$
I^{\prime \prime}=\gamma I^{\prime}
$$

kann (1) durch folgenden Zusammenhang ausgedrückt werden:

$$
I_{i}=I_{\text {sat }} \beta\left[1+\gamma \cos \left(\varphi+\psi_{i}\right)\right]
$$

Hierin bezeichnet $I_{\text {sat }}$ die Sättigungskapazität des Pixels, $\beta$ die relative mittlere Intensität (Belichtung) und $\gamma$ den relativen Streifenkontrast (Sichtbarkeit). Die beiden Parameter $\beta$ und $\gamma$ beschreiben die Kontrastverhältnisse des Musters im betrachteten Pixel unabhängig vom Wertebereich der Kamera. Beide weisen einen Wertebereich von 0 bis 1 auf, wobei zusätzlich die Bedingung:

$$
\beta \leq \frac{1}{1+\gamma}
$$

gilt, da die Intensität $I_{i}$ im Pixel sonst höher wäre als die Sättigungskapazität $I_{\text {sat }}$. In Abbildung 2 ist die Wirkung von $\beta$ und $\gamma$ auf den Streifenkontrast visualisiert. Für $\beta \geq 0,5$ sind die gültigen Werte gemäß (5) beschränkt. Der optimale Streifenkontrast wird für $\beta=0,5$ und $\gamma=1$ erreicht.

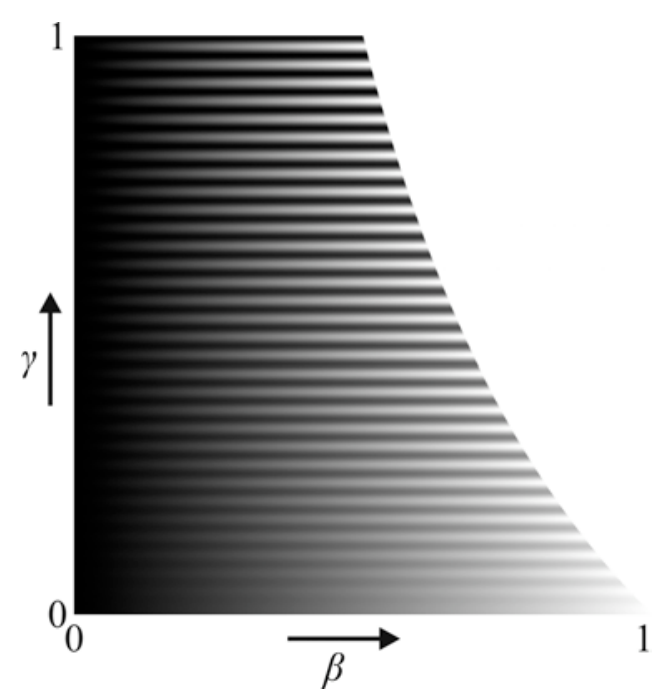

Abbildung 2: Streifenmuster in Abhängigkeit von $\beta$ und $\gamma$

Der Vorteil der Darstellung gemäß (4) ist neben der Normierung der beschreibenden Parameter $\beta$ und $\gamma$ insbesondere die Trennung von skalierenden und modulierenden Einflüssen. Die Belichtung $\beta$ wird dabei im Wesentlichen durch Effekte festgelegt, welche die auf den Sensor gelangende Lichtmenge unabhängig von der Modulation beeinflussen, wie z.B.: 
- die maximale Helligkeit des Projektors bzw. Displays,

- die Belichtungszeit und die Blendeneinstellung der Kamera,

- den Lichtverlust durch optische Elemente (z.B. Filter),

- die Streucharakteristik des Messobjekts bzw. des Displays.

Die Sichtbarkeit der Streifen $\gamma$ hingegen wird beeinflusst durch:

- den maximalen Kontrast des Projektors bzw. Displays,

- den relativen Anteil von Störlicht im Signal,

- die Kontrastübertragungsfunktion der optischen Elemente,

- die Schärfentiefe.

Nach der Aufzeichnung der Bildsequenz kann aus den beobachteten Intensitäten $I_{1}, \ldots, I_{M}$ durch Anwendung geeigneter Phasenschiebe-Algorithmen für jedes Kamerapixel unabhängig von seiner Nachbarschaft ein Phasenwert ermittelt werden, indem das Gleichungssystem (4) nach $\varphi$ aufgelöst wird. Der Einfluss des Intensitätsrauschens auf die zufälligen Phasenabweichungen wurde von Rathjen [2] allgemeingültig auf der Grundlage bivariater Normalverteilungen analysiert. Die wichtigsten Ergebnisse dieser Arbeit sind in den folgenden Abschnitten wiedergegeben.

Ausgehend von einer allgemeinen Beschreibung eines Phasenschiebe-Algorithmus in Form eines von den Intensitäten $I_{i}$ abhängigen Bruches mit einem Zähler $Z$ und einem Nenner $N$ :

$$
\tan \varphi=\frac{Z\left(I_{1}, \ldots, I_{M}\right)}{N\left(I_{1}, \ldots, I_{M}\right)}
$$

konnte gezeigt werden, dass die Form der resultierenden Verteilungsdichtefunktion für den Phasenwinkel $\varphi$ neben dem Signal-Rausch-Verhältnis $(S N R)$ im Wesentlichen vom Korrelationskoeffizient $\rho$ und dem Verhältnis $\alpha$ der Standardabweichungen von Zähler $\sigma_{Z}$ und Nenner $\sigma_{N}$ abhängt. Unter Verwendung der Kovarianz von Zähler und Nenner $\sigma_{N Z}$ gilt dabei:

$$
\rho=\frac{\sigma_{N Z}}{\sigma_{N} \sigma_{Z}}
$$

und

$$
\alpha=\frac{\sigma_{Z}}{\sigma_{N}}
$$

Als Ergebnis der Analyse zeigte sich:

1. Aufgrund des begrenzten Wertebereichs der Arkustangensfunktion ist der Phasenwinkel $\varphi$ nicht normalverteilt. Nur für $S N R \gg>1$ kann in guter Näherung von einer Normalverteilung ausgegangen werden.

2. Im Allgemeinen Fall ist die Verteilungsdichtefunktion schief und $2 \varphi$-abhängig von der beobachteten Phasenlage.

3. Nur für $\rho=0$ und $\alpha=1$ ist die Verteilungsdichtefunktion phasenunabhängig und der Phasenschiebe-Algorithmus ein erwartungstreuer Schätzer für den unbekannten Phasenwinkel.

Diese Ergebnisse decken sich mit der Analyse von Bothe [7] bei ähnlichen Untersuchungen von Phasenauswertungen. Aufgrund der in Abschnitt 1 erläuterten Relevanz stochastischer Abweichungen für Messverfahren mit strukturierter Beleuchtung sind viele der in der Interferometrie eingesetzten Algorithmen hierfür ungeeignet, da sie zwar systematische Fehlereinflüsse minimieren, aber bezüglich ihrer statistischen Eigenschaften $(\rho \neq 0$ und / oder $\alpha \neq 1$ ) ein ungünstiges Verhalten zeigen. 
Eine Gruppe von Phasenschiebe-Algorithmen die hingegen beide Bedingungen erfüllen, sind symmetrische M-Schritt Algorithmen. Diese basieren auf gleichmäßig über eine volle Periode verteilten Phasenverschiebungen:

$$
\tan \varphi=\frac{-\sum_{i=1}^{M} I_{i} \sin \psi_{i}}{\sum_{i=1}^{M} I_{i} \cos \psi_{i}} ; \quad \psi_{i}=\frac{i 2 \pi}{M}
$$

mit dem bekannten 4-Schritt Algorithmus für $M=4$ :

$$
\tan \varphi=\frac{I_{3}-I_{1}}{I_{4}-I_{2}} ; \quad \psi_{i}=\left(\frac{\pi}{2} ; \pi ; \frac{3 \pi}{2} ; 2 \pi\right)
$$

Aufgrund der positiven statistischen Eigenschaften beschränkt sich die weitere Analyse daher auf diese Klasse von Phasenschiebe-Algorithmen. Gemäß [3] kann hierfür ein einfacher Zusammenhang zwischen dem Intensitätsrauschen des Bildsensors $\sigma_{I}$ und dem Phasenrauschen $\sigma_{\varphi}$ angegeben werden:

$$
\sigma_{\varphi}=\sqrt{\frac{2}{M}} \frac{1}{\gamma} \frac{\sigma_{I}}{I^{\prime}}=\sqrt{\frac{2}{M}} \frac{1}{\gamma} \frac{1}{S N R}
$$

Dieser Ausdruck basiert auf der Annahme von signalunabhängigem normalverteiltem Intensitätsrauschen mit konstantem $\sigma_{I}$, wodurch das Signal-Rausch-Verhältnis linear mit der mittleren Intensität $I^{\prime}$ steigt. Im folgenden Abschnitt 3 wird gezeigt, dass (11) auf der Grundlage eines komplexeren Kameramodells weiterentwickelt werden kann.

\section{Herleitung des Phasenrauschmodells}

Ausgangspunkt der folgenden Ausführungen war die Fragestellung, inwieweit der einfache Zusammenhang (11) für symmetrische M-Schritt Phasenschiebe-Algorithmen auch gilt, wenn statt konstantem Intensitätsrauschen ein komplexeres Kameramodell angenommen wird. Wie in Abschnitt 1 erläutert, ist ein solches allgemein anerkanntes Modell in der Norm EMVA 1288 die Grundlage für die objektive Charakterisierung von Bildsensoren und Kameras. Neben dem mathematischen Modell sind in der Norm auch experimentelle Verfahren zur Bestimmung der Modellparameter angegeben. Das Modell umfasst dabei zeitliche und räumliche Rauscheffekte ebenso wie eine detaillierte Berücksichtigung thermischer Effekte in Form des temperaturabhängigen Dunkelstroms. Da bei den hier betrachteten PhasenschiebeAlgorithmen die Auswertung in einem Pixel unabhängig von benachbarten Pixeln erfolgen und der Einfluss des Dunkelstroms bei diesen Anwendungen im Allgemeinen vernachlässigt werden kann, beschränkt sich die weitere Analyse auf das zeitliche Rauschmodell der EMVA 1288.
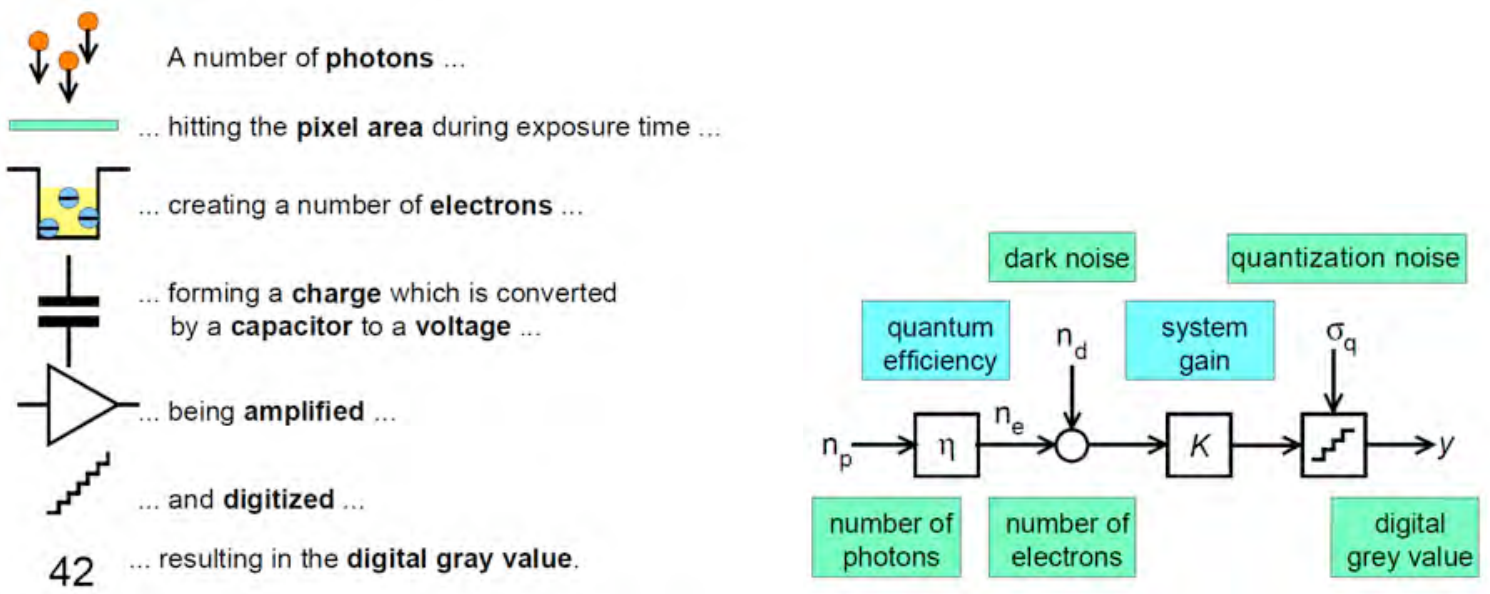

Abbildung 3: Bildentstehung (links) und mathematisches Kameramodell (rechts) (Quelle: EMVA 1288) 
Der im Kameramodell zugrunde gelegte physikalische Prozess der Bildentstehung ist auf der linken Seite von Abbildung 3 dargestellt. Er entspricht im Wesentlichen dem Belichtungs- und Ausleseprozess eines CCD-Elements. Das Modell kann aber gemäß [6] auch für CMOS-Sensoren verwendet werden, wenn sie über eine lineare Kennlinie verfügen. Das zugehörige mathematische Modell (Abbildung 3 rechts) modelliert diesen Prozess als multiplikative Umrechnung der Anzahl Photonen $n_{p}$ in die Anzahl der Signalelektronen $n_{e}$, mit der Quanteneffizienz $\eta$ als Faktor, und weiter in den digitalen Grauwert $y$ mit der Systemverstärkung $K$ als Faktor. Drei Rauschquellen werden dabei zusätzlich berücksichtigt:

- die erzeugten Elektronen sind Poisson-verteilt $\left(\sigma_{e}^{2}=\mu_{e}\right)$

- das additive elektronische Ausleserauschen ist normalverteilt $\left(\sigma_{d}, \mu_{d}\right)$

- die Quantisierung erzeugt gleichverteiltes Rauschen im Bereich $\pm 1 / 2$ digitale Grauwerte $\left(\sigma_{q}{ }^{2}=1 / 12\right)$

Der gesamte Prozess wird als linear angenommen, so dass sich die Varianz des Ausgangssignals $\sigma_{y}$ durch Addition der Einzelvarianzen unter Berücksichtigung der Systemverstärkung ergibt:

$$
\sigma_{y}^{2}=K^{2}\left(\sigma_{e}^{2}+\sigma_{d}^{2}\right)+\sigma_{q}^{2}
$$

Auf der Grundlage von (12) wird das Signal-Rausch-Verhältnis für das Ausgangssignal $y$ definiert als das Verhältnis zwischen der Differenz $\left(\mu_{y}-\mu_{y . d a r k}\right)$ der Erwartungswerte von Ausgangssignal und Dunkelrauschen und der Standardabweichung des Ausgangssignals $\sigma_{y}$, wobei alle Größen mit dem Index $y$ jeweils die Einheit „digitaler Grauwert" (DN = digital number) besitzen:

$$
S N R=\frac{\mu_{y}-\mu_{y . d a r k}}{\sigma_{y}}=\frac{\eta \mu_{p}}{\sqrt{\eta \mu_{p}+\sigma_{d}^{2}+\frac{1}{12} \frac{1}{K^{2}}}} .
$$

Es ist erkennbar, dass die Beleuchtungsstärke $\mu_{p}$ aufgrund der Poisson-Verteilung auch unter der Wurzel im Ausdruck für $\sigma_{y}$ vorhanden ist, so dass das Intensitätsrauschen signalabhängig wird. Für kleine Werte von $\mu_{p}$ ist das Signal-Rausch-Verhältnis daher proportional zur Beleuchtungsstärke und es dominiert der Einfluss des Ausleserauschens. Für große Werte von $\mu_{p}$ ist es proportional zur Wurzel aus der Beleuchtungsstärke und wird vom Photonenrauschen dominiert.

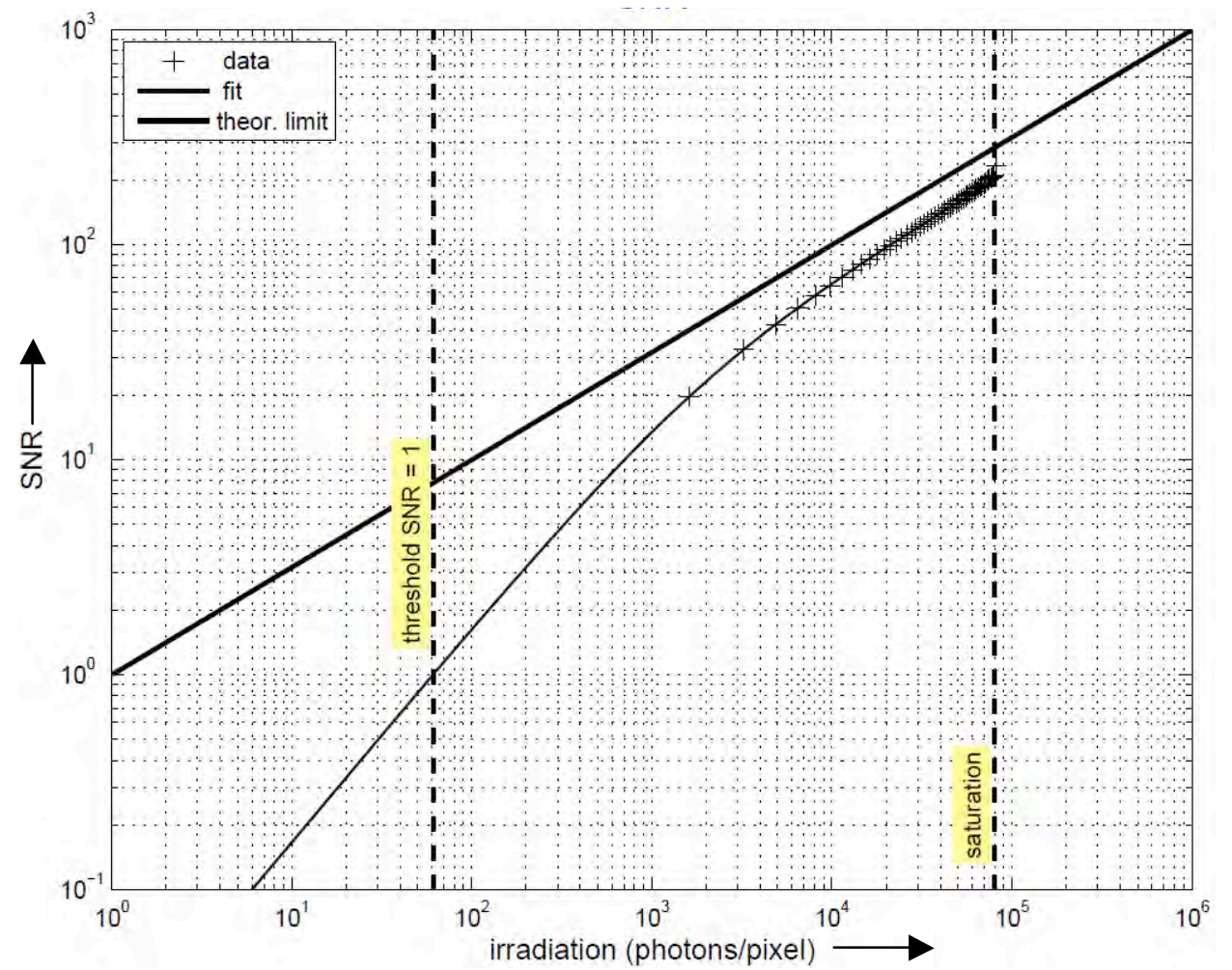

Abbildung 4: SNR-Diagramm in einem EMVA 1288 konformen Datenblatt (Quelle: EMVA 1288) 
In einem EMVA 1288 konformen Datenblatt wird der Verlauf des Signal-Rausch-Verhältnisses über der Beleuchtungsstärke doppelt-logarithmisch dargestellt (vgl. Abbildung 4). Die beiden beschriebenen dominierenden Effekte sind dann sehr gut als Asymptoten mit der Steigung 1 für kleine Beleuchtungsstärken und mit der Steigung $1 / 2$ für große Beleuchtungsstärken erkennbar. Zusätzlich ist das theoretische Limit, welches durch die Poisson-Statistik festgelegt ist, als Gerade mit dem Anfangspunkt $(1,1)$ und der Steigung $1 / 2$ eingezeichnet. Dies entspricht dem $S N R$-Verlauf einer idealen Kamera $\left(\eta=1, \sigma_{q}=\sigma_{d}=0\right)$ und ermöglicht eine Bewertung, wie weit die spezifizierte Kamera am physikalischen Limit betrieben werden kann. Bei der Interpretation eines SNR-Diagramms ist jedoch zu beachten, dass je nach Anwendung unterschiedliche Bereiche der Kurve relevant sind. So kann man beim Einsatz von optischen Messsystemen mit strukturierter Beleuchtung davon ausgehen, dass die minimal geforderte Belichtung mindestens $10 \%$ der Sättigungskapazität beträgt, so dass in diesen Anwendungen das Intensitätsrauschen typischerweise vom Photonen-Rauschen dominiert wird.

Ein Modell für die stochastischen Abweichungen bei der Anwendung von symmetrischen M-Schritt Phasenschiebealgorithmen kann durch Kombination der in den vorangegangenen Abschnitten dargestellten Ergebnisse hergeleitet werden. Im Ausdruck für das Signal-Rausch-Verhältnis (13) kann in Anlehnung an (4) ein Bezug zur Belichtung $\beta$ und der Sättigungskapazität $I_{\text {sat }}$ (hier Elektronensättigungskapazität $\mu_{\text {e.sat }}$ ) hergestellt werden durch den Zusammenhang:

$$
\eta \mu_{p}=\mu_{e}=\beta \mu_{e . s a t}
$$

Durch Einsetzen in den Ausdruck für das Phasenrauschen bei symmetrischen M-Schritt Algorithmen (11) ergibt sich eine Formel zur Vorhersage des Phasenrauschens, welche sowohl Auslese-, als auch Photonen- und Quantisierungsrauschen berücksichtigt:

$$
\sigma_{\varphi}=\sqrt{\frac{2}{M}} \frac{1}{\gamma \beta \mu_{\text {e.sat }}} \sqrt{\beta \mu_{\text {e.sat }}+\sigma_{d}^{2}+\frac{1}{12 K^{2}}} .
$$

Hierin sind alle Parameter entweder fundamentale Größen welche die Messung beschreiben $(\beta, \gamma, M)$ oder Kameraparameter die einem EMVA 1288 konformen Datenblatt entnommen werden können $\left(\mu_{e . s a t}, \sigma_{d}, K\right)$.

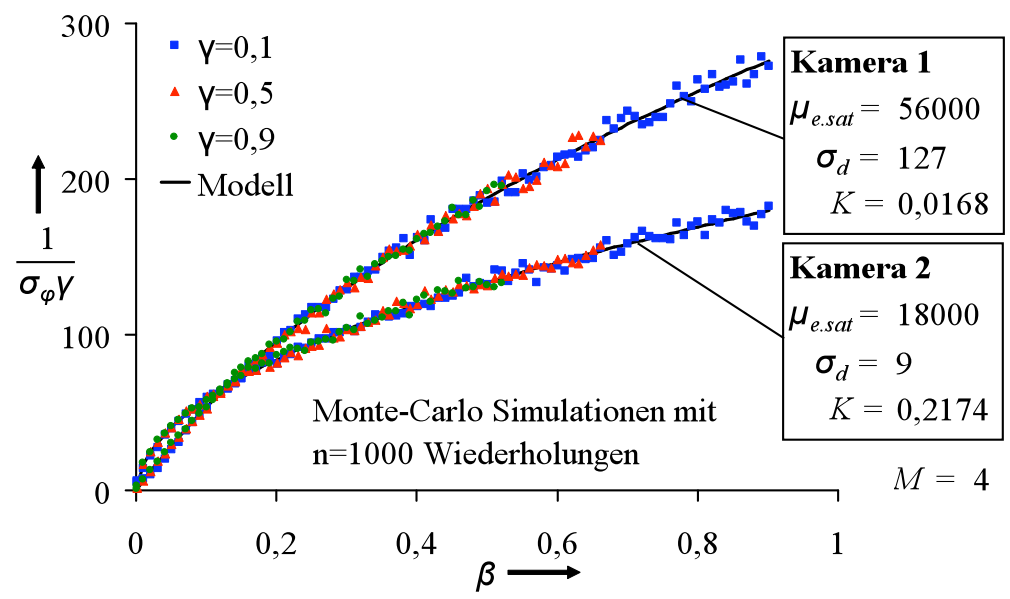

Abbildung 5: Ergebnisse von Monte-Carlo-Simulationen zur Validierung des Phasenrauschmodells

Das Phasenrauschmodell wurde sowohl durch Monte-Carlo-Simulationen als auch experimentell validiert. Für die Simulationen wurde der physikalische Bildentstehungsprozess (vgl. Abbildung 3 links) nachgebildet, wobei der diskrete Charakter der Signalgrößen in Form ganzzahliger Variablen berücksichtigt wurde. Weitere nichtlineare Effekte wie z.B. Kennlinienabweichungen beim Auslesevorgang sind vernachlässigt. In Abbildung 5 sind beispielhaft Simulationsergebnisse für zwei verschiedene Kameras dargestellt. Die Abhängigkeit von der Sichtbarkeit $\gamma$ wurde dabei durch Berücksichtigung auf der Ordinatenachse eliminiert. Dadurch liegen die Punkte für beliebige Werte von $\gamma$ auf der gleichen Modellkurve. Zur Erhöhung der Lesbarkeit ist der Kehrwert des Phasenrauschens dargestellt, wodurch der Verlauf der Modellkurve annähernd wurzelförmig ist. Es ist erkennbar, dass sich das physikalische Modell über einen weiten Parameterbereich sehr gut durch (15) abbilden lässt. Lediglich für sehr geringe Werte von $\beta$ und $\gamma$ kleiner als 0,1 sind systematische Abweichungen beobachtbar. Diese sind jedoch mit dem daraus resultierenden 
sehr kleinen SNR und der damit verbundenen Abweichung der Verteilungsdichtefunktion von einer Normalverteilung erklärbar. Zusätzlich ist dieser Parameterbereich, aufgrund der dann zu erwartenden sehr großen stochastischen Abweichungen, für messtechnische Anwendungen nicht relevant.

Für die Experimente wurde ein LCD-Monitor von der untersuchten Kamera beobachtet. Auf dem Display erfolgte die Darstellung mehrerer Muster für verschiedene Ausprägungen von $\beta$ und $\gamma$ mit einer anschließenden statistischen Auswertung von jeweils 1000 Phasenberechnungen. Die Ergebnisse der Untersuchungen (vgl. Abbildung 6) zeigen eine deutlich bessere Übereinstimmung zwischen der Modellvorhersage und Messdaten als das Modell (11), das einen linearen Verlauf für den dargestellten Fall vorhersagt.

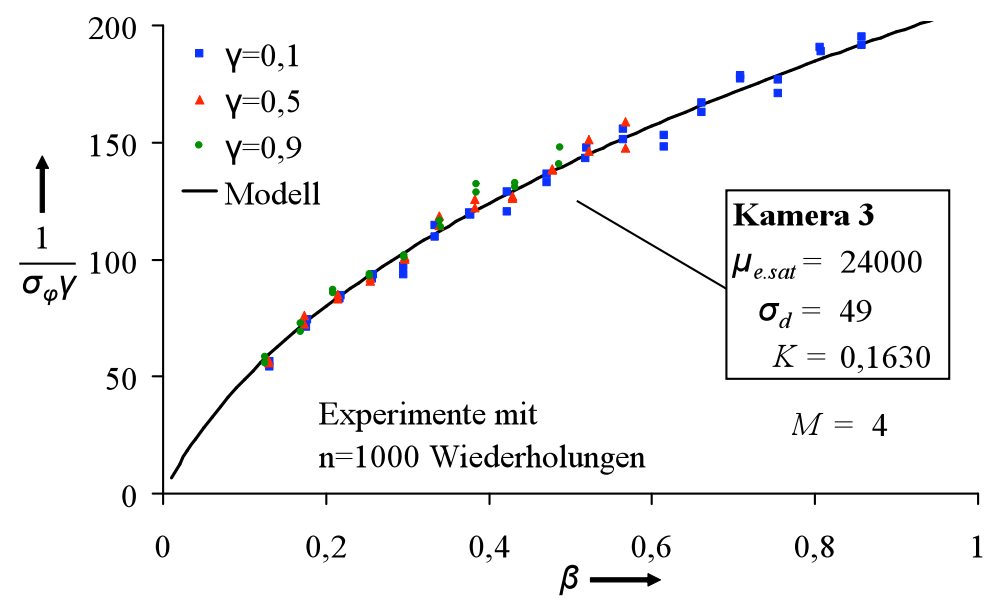

Abbildung 6: Ergebnisse von Experimenten zur Validierung des Phasenrauschmodells

Da nicht bei allen Herstellern von Kameras und Bildsensoren EMVA 1288 konforme Datenblätter bereit gestellt werden, steht der Anwender in diesen Fällen vor dem Problem, die Modellparameter selbst bestimmen zu müssen. Prinzipiell können dafür die in der Norm beschriebenen experimentellen Verfahren eingesetzt werden. Dabei wird der Sensor der Kamera ohne Objektiv homogen beleuchtet und das Intensitätsrauschen bei verschiedenen Belichtungsstufen jeweils über alle Pixel eines Differenzbildes bestimmt. Dadurch werden ortsabhängige Offsetfehler eliminiert und man benötigt lediglich zwei Aufnahmen pro Belichtungsstufe. In der praktischen Realisierung ist dieses Verfahren jedoch gerätetechnisch aufwendig, da es sehr empfindlich gegenüber Beleuchtungsinhomogenitäten ist.

Das hier vorgeschlagene alternative Verfahren umgeht dieses Problem durch die statistische Auswertung von Wiederholungsmessungen. Dadurch erhöht sich zwar deutlich die Messzeit zur Ermittlung der Parameter, allerdings ist dies nur einmalig notwendig und die Messungen können ohne zusätzlichen Geräteaufwand direkt mit dem Messsystem durchgeführt werden. Die weitere Auswertung ist jedoch identisch zu der in der EMVA 1288 beschriebenen Photon-Transfer-Methode. Zunächst werden dabei in einer Messung mit abgedecktem Objektiv der zeitliche Mittelwert der Grauwerte $\mu_{v . d a r k}$ und die Grauwertvarianz des Dunkelrauschens $\sigma_{y . d a r k}^{2}$ ermittelt. Dann werden mit Hilfe des Projektors bzw. des Displays mehrere einfarbige Bilder mit unterschiedlicher Helligkeit angezeigt und ebenfalls jeweils der mittlere Grauwert $\mu_{y}$ und die Grauwertvarianz $\sigma_{y}^{2}$ bestimmt. Wird nun in einem Diagramm jeweils die Differenz $\left(\sigma_{y}^{2}-\sigma_{y \text { dark }}^{2}\right)$ über der Differenz $\left(\mu_{y}-\mu_{y, d a r k}\right)$ aufgetragen (vgl. Abbildung 7), so entspricht die Steigung des linearen Teils der Kurve gerade dem gesuchten Parameter $K$. In der Norm wird für den linearen Fit ein Bereich vom minimalen Wert bis zu $70 \%$ der Sättigungskapazität $\mu_{y \text { sat }}$ festgelegt. Die Sättigungskapazität entspricht dabei dem Grauwert, bei dem die Grauwertvarianz $\sigma_{y}^{2}$ ein Maximum erreicht.

Die Umrechnung auf die bei den Modellparametern verwendete Einheit (Anzahl der Elektronen) erfolgt dann gemäß:

$$
\mu_{d}=\frac{\mu_{y . d a r k}}{K} \quad, \quad \mu_{e . s a t}=\frac{\mu_{y . s a t}}{K} \quad, \quad \sigma_{d}=\frac{\sqrt{\sigma_{y . d a r k}^{2}-\frac{1}{12}}}{K} .
$$

Auf diese Weise lassen sich alle für das Phasenrauschmodell benötigten Parameter für jedes Pixel unabhängig von der Beleuchtungshomogenität ermitteln. Aufgrund der zu erwartenden statistischen Schwankungen bietet sich anschließend eine Mittelung der Werte bzw. bei erkennbarer Systematik die Anpassung einer ortsabhängigen Parameterfunktion an. 


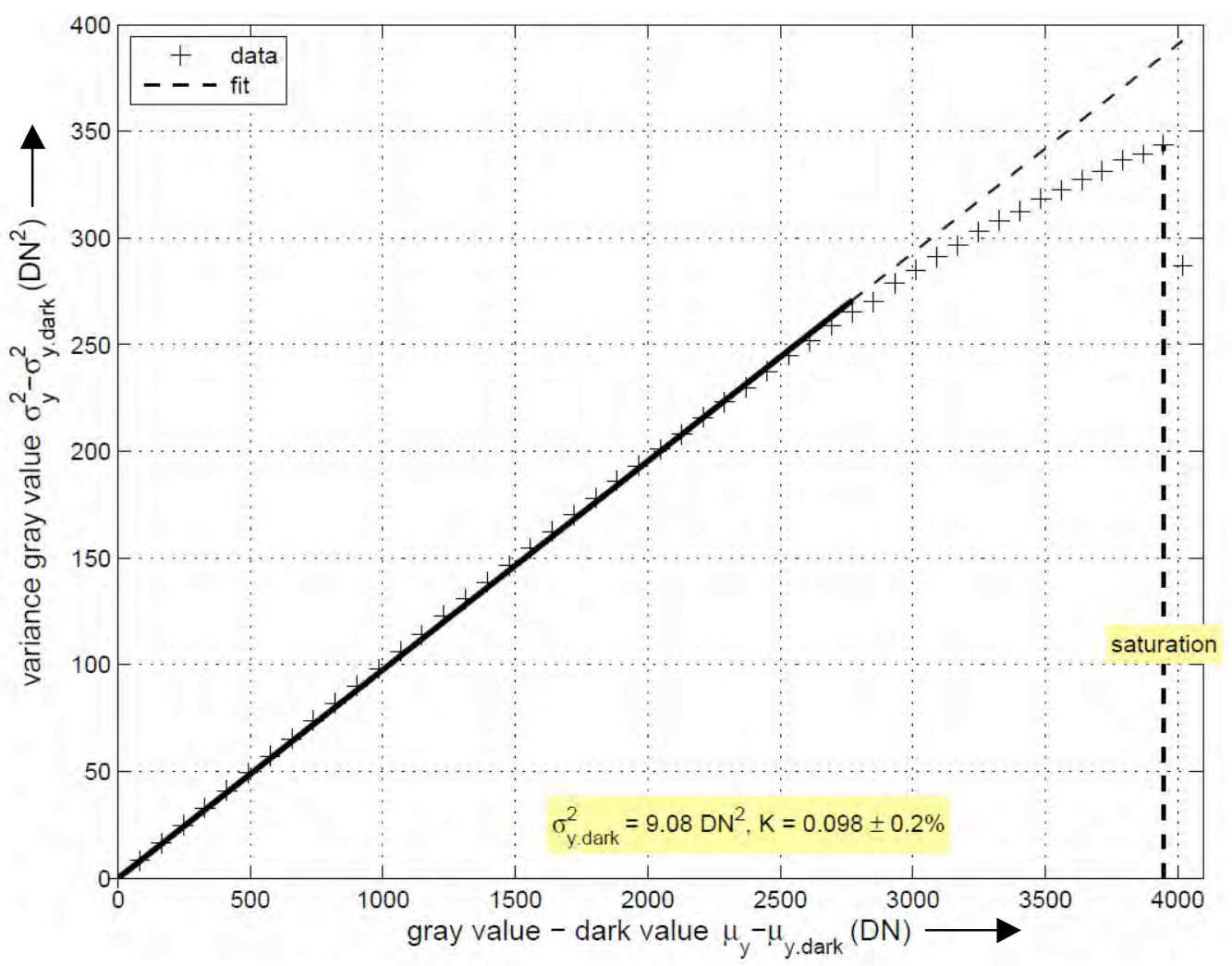

Abbildung 7: Photon-Transfer-Methode zur Ermittlung von K (Quelle: EMVA 1288)

\section{Methode zur Vorhersage des Phasenrauschens}

Die für eine Vorhersage des Phasenrauschens gemäß (15) benötigten Kameraparameter $\left(\mu_{e . s a t}, \sigma_{d}, K\right)$ können einem EMVA 1288 konformen Datenblatt entnommen oder nach dem in Abschnitt 3 beschriebenen Vorgehen experimentell ermittelt werden. Dies ermöglicht bereits die vergleichende Bewertung verschiedener Kameras und die allgemeine Beurteilung in welchem Parameterbereich eine Kamera die an das Messsystem gestellte Genauigkeitsanforderung erfüllen kann. Dies ist beispielhaft in Abbildung 8 in Form eines Phasenrauschdiagramms demonstriert. In dem Diagramm sind die Linien gleichen Phasenrauschens $\sigma_{\varphi}$ über den beiden Parametern $\gamma$ und $\beta$ eingezeichnet. Das Phasenrauschen ist dabei jeweils als Bruchteil der Wellenlänge des Musters angegeben. Als typischer Wert für die Genauigkeit von Phasenschiebe-Auswertungen wird häufig /100 angegeben und es ist erkennbar, dass die untersuchte Kamera diese Genauigkeit bei Anwendung des 4-Schritt-Algorithmus über einen weiten Parameterbereich erreicht. Das theoretische Limit für diese Kamera liegt bei etwa $/ 850$, realistisch zu erwartende Werte bei kooperativen Oberflächen liegen zwischen /300 und /500.

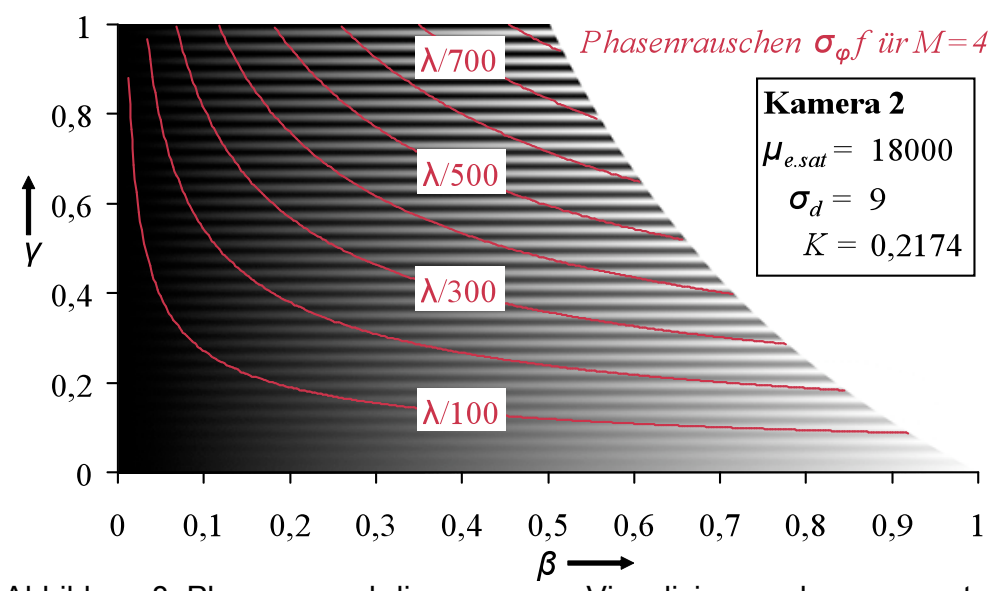

Abbildung 8: Phasenrauschdiagramm zur Visualisierung der zu erwartenden stochastischen Abweichungen 
Neben den Kameraparametern werden in (15) zur Vorhersage des Phasenrauschens auch die Messparameter $(\beta, \gamma, M)$ benötigt. Während $\mathrm{M}$ bereits durch die Wahl des Auswertealgorithmus festgelegt ist, hängen $\beta$ und $\gamma$ in einer konkreten Messung auch vom Messobjekt selbst ab und können daher nicht als a priori bekannte konstante Parameter angesetzt werden. Es ist jedoch möglich, das Gleichungssystem (1) neben dem Phasenwinkel $\varphi$ auch nach den anderen unbekannten Größen $I^{\prime}$ und $I^{\prime \prime} z u$ lösen. Für den symmetrischen M-Schritt Algorithmus gilt dabei nach [1]:

$$
I^{\prime}=\frac{1}{M} \sum_{i=1}^{M} I_{i}
$$

und

$$
I^{\prime \prime}=\frac{2}{M} \sqrt{\left(\sum_{i=1}^{M} I_{i} \sin \psi_{i}\right)^{2}+\left(\sum_{i=1}^{M} I_{i} \cos \psi_{i}\right)^{2}}
$$

Diese Gleichungen können mit (2) und (3) zu Ausdrücken für die aus den aufgezeichneten Intensitäten geschätzten Parameter $\beta_{\text {est }}$ und $\gamma_{\text {est }}$ kombiniert werden:

$$
\beta_{\text {est }}=\frac{I^{\prime}}{I_{\text {sat }}}=\frac{\frac{1}{M} \sum_{i=1}^{M} I_{i}}{K \mu_{\text {e.sat }}}
$$

und

$$
\gamma_{e s t}=\frac{I^{\prime \prime}}{I^{\prime}}=2 \frac{\sqrt{\left(\sum_{i=1}^{M} I_{i} \sin \psi_{i}\right)^{2}+\left(\sum_{i=1}^{M} I_{i} \cos \psi_{i}\right)^{2}}}{\sum_{i=1}^{M} I_{i}} .
$$

Für die weitere Analyse muss beachtet werden, dass die aufgezeichneten Intensitäten $I_{i}$ in (19) und (20) nicht direkt durch die von der Kamera ausgegebenen Grauwerte $y_{i}$ repräsentiert werden, da gemäß (13) das Dunkelrauschen $\mu_{y \text {.dark }}$ vor der Weiterverarbeitung subtrahiert werden muss. Wenn diese Korrektur vorgenommen wurde, kann (19) und (20) in (15) eingesetzt und zu einem Ausdruck für das auf der Grundlage der aufgezeichneten Grauwerte geschätzte Phasenrauschen $\sigma_{\varphi . e s t}$ vereinfacht werden zu:

$$
\sigma_{\varphi . e s t}=\sqrt{\frac{\frac{K}{2} \sum_{i=1}^{M} y_{i}+\frac{K^{2} M}{2}\left(\sigma_{d}^{2}-\mu_{d}\right)+\frac{M}{24}}{\left(\sum_{i=1}^{M} y_{i} \sin \psi_{i}\right)^{2}+\left(\sum_{i=1}^{M} y_{i} \cos \psi_{i}\right)^{2}}} .
$$

Die Abhängigkeit von der Sättigungskapazität $\mu_{\text {e.sat }}$ wurde dabei eliminiert, was die in Abschnitt 3 beschriebenen Ermittlung der benötigten Parameter weiter vereinfacht, da insbesondere eine genaue Bestimmung von $\mu_{\text {e.sat }}$ nur mit einer hohen Messpunktdichte in der Nähe der Sättigung möglich ist.

Die Genauigkeit der Vorhersage, d.h. die stochastischen Abweichungen des mit (21) geschätzten Phasenrauschens, folgt direkt aus den Gesetzen der Fehlerfortpflanzung:

$$
\sigma\left(\sigma_{\varphi . e s t}\right)=\sigma_{\varphi}^{2}
$$

Das bedeutet, dass die relative Vorhersagegenauigkeit gerade dem Wert von $\sigma_{\varphi}$ entspricht. Für Messsysteme mit strukturierter Beleuchtung kann daher von einer relativen statistischen Unsicherheit der Schätzung von $<6 \%$, entsprechend einem Phasenrauschen von $</ 100$, ausgegangen werden. 
Die oben beschriebene Methode zur Vorhersage des Phasenrauschens aus den aufgezeichneten Grauwerten wurde ebenfalls experimentell validiert. Die Experimente wurden dabei analog $\mathrm{zu}$ der in Abschnitt 3 beschriebenen Validierung des Phasenrauschmodells durchgeführt, d.h. es wurden Muster mit unterschiedlichen Parameterausprägungen angezeigt und wiederholt ausgewertet. Im oberen Diagramm in Abbildung 9 ist für jeweils 1000 Wiederholungen die Verteilung des für jede Messung individuell geschätzten $\sigma_{\varphi . e s t}$ über den aus allen Phasenmessungen statistisch ermittelten $\sigma_{\varphi}$ aufgetragen. Es ist erkennbar, dass das Modell sowohl bezüglich des Mittelwerts als auch bezüglich der erwarteten Streuung der Werte sehr gut mit den Messungen übereinstimmt. Im unteren Diagramm ist zusätzlich die Gültigkeit von (22) demonstriert, indem die relative Standardabweichung der geschätzten Werte über dem Phasenrauschen aufgetragen ist.
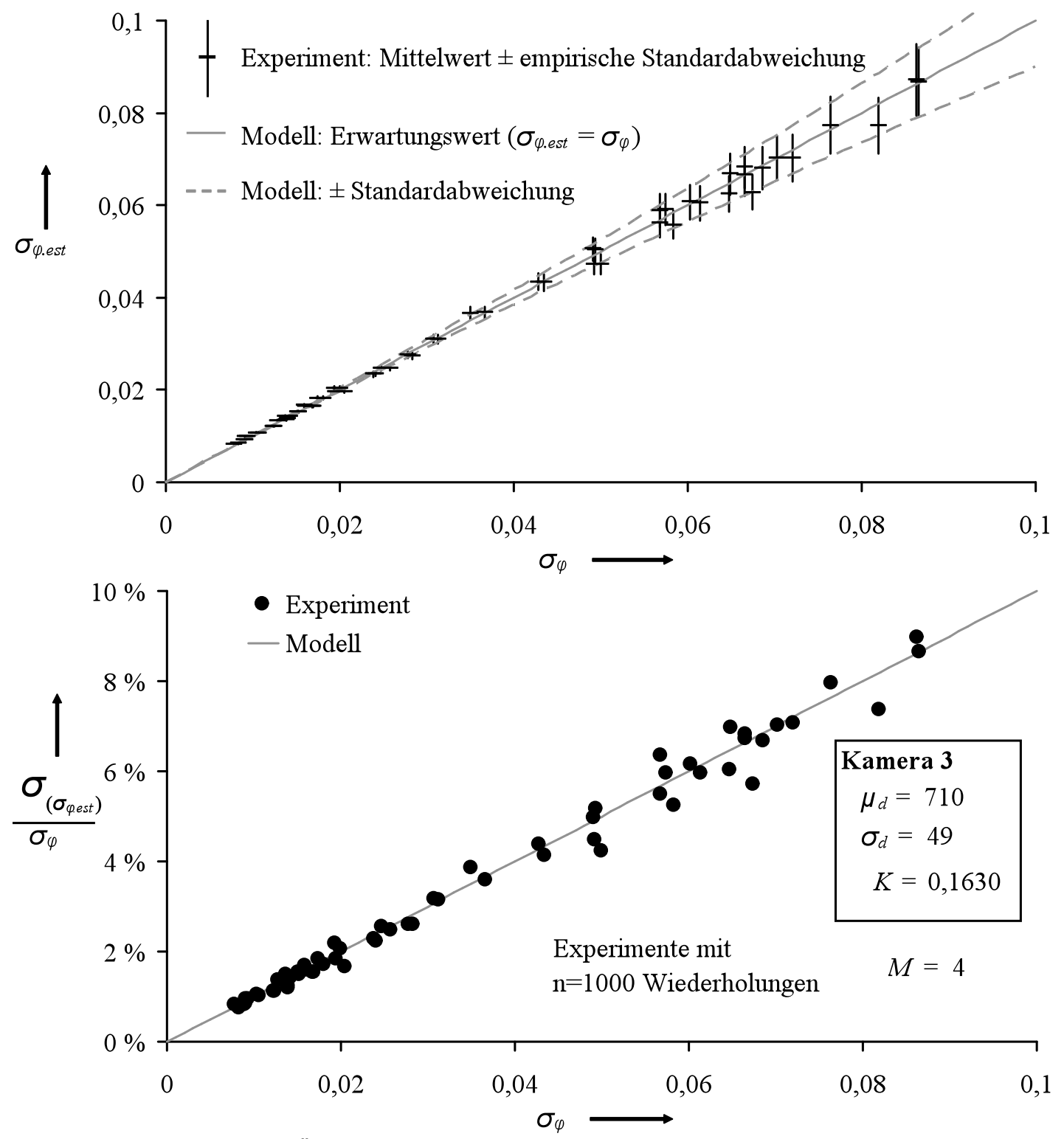

Abbildung 9: Experimentelle Überprüfung der Methode zur Vorhersage des Phasenrauschens

Die durchgeführten Experimente lassen den Schluss zu, dass das beschriebene Verfahren mit hinreichender Genauigkeit in der Lage ist, den gewünschten Schätzwert für die stochastische Abweichung des Phasenwerts für jedes Pixel aus den aufgenommenen Streifenbildern zu berechnen. Dieser Wert kann dann in einem optischen Messsystem mit strukturierter Beleuchtung zum Einen direkt als Qualitätsmaß zur Maskierung ungültiger Messwerte verwendet werden. Zum Anderen steht er für die Weiterverarbeitung als Gewichtungsfaktor zur Verfügung. So kann beispielsweise in einer Messung mit einem Streifenprojektionssystem das aus der Triangulation resultierende Konfidenzellipsoid für jeden 3D-Punkt unabhängig ermittelt werden, ohne dass dafür aufwendige Wiederholungsmessungen notwendig sind. 


\section{Zusammenfassung}

Ausgangspunkt der vorliegenden Arbeit war die Fragestellung, ob es möglich ist, in einem optischen Messsystem mit strukturierter Beleuchtung bei Verwendung von phasenverschobenen sinusförmigen Streifenmustern einen Wert für die stochastischen Abweichungen der Ortsinformation zu berechnen ohne aufwendige Wiederholungsmessungen durchführen zu müssen. Es wurde anhand der statistischen Eigenschaften von Phasenschiebe-Algorithmen gezeigt, dass dies für symmetrische M-Schritt Algorithmen möglich ist und dass diese Klasse von Algorithmen auch die besten Ergebnisse bezüglich des Phasenrauschens liefert. Vorraussetzung für die Berechnung eines Schätzwertes der stochastischen Phasenabweichungen für jeden Messwert ist die Verfügbarkeit eines hinreichend genauen Kamera-Rauschmodells sowie der dafür notwendigen Modellparameter. Mit der Norm EMVA 1288 steht ein solches Modell zur Verfügung und die Parameter können entweder einem konformen Kameradatenblatt entnommen oder mit der in dieser Arbeit beschriebenen experimentellen Methode ermittelt werden. Das auf der Grundlage dieses Kameramodells entwickelte Phasenrauschmodell wurde mittels Monte-Carlo-Simulationen und Experimenten validiert. Es konnte gezeigt werden, dass das Modell innerhalb des typischen Parameterbereichs bei Messsystemen mit strukturierter Beleuchtung die statistischen Eigenschaften der Phasenauswertung sehr gut abbildet. Darüber hinaus wurde auf der Grundlage des Phasenrauschmodells eine Methode entwickelt, welche eine Vorhersage des Phasenrauschens während einer Messung direkt aus den aufgezeichneten Grauwerten ermöglicht. Die Gültigkeit dieser Methode wurde ebenfalls experimentell demonstriert und es konnte gezeigt werden, dass die relative Genauigkeit dieser Vorhersagemethode, für die in praktischen Anwendungen typischerweise erreichbaren stochastischen Phasenabweichungen von kleiner als 1100 , geringer als $6 \%$ ist. Der so berechnete Schätzwert für das Phasenrauschen steht dann für jeden Messpunkt zur Verfügung und kann für die weitere Verarbeitung der Messdaten, z.B. als Gewichtungsfaktor bei der Anpassung geometrischer Standardelemente oder bei der Fusion mehrerer Messungen, eingesetzt werden. Aktuelle Weiterentwicklungen des Verfahrens zielen darauf ab, auch bei der experimentellen Ermittlung der Modellparameter auf Wiederholungsmessungen verzichten zu können.

\section{Danksagung}

Die Autoren danken der Deutschen Forschungsgemeinschaft (DFG) für die Förderung dieser Arbeit im Rahmen des Projekts Pe1402/2-1.

\section{Literatur}

[1] Schreiber, H.; Bruning, J. H.: Phase Shifting Interferometry. Malacara, D. (Hrsg.): Optical Shop Testing. 3rd ed., Hoboken, NY: Wiley, S. 547-655, 2007

[2] Rathjen, C.: Statistical properties of phase-shift algorithms. Journal of the Optical Society of America A, 12 (1995) 9, S. 1997-2008

[3] Surrel, Y.: Additive noise effect in digital phase detection. Applied Optics 36 (1997) 1, S. 271-276

[4] Hibino, K.: Susceptibility of systematic error-compensating algorithms to random noise in phaseshifting interferometry. Applied Optics 36 (1997) 10, S. 2084-2093

[5] Notni, G. H.; Notni, G.: Digital fringe projection in 3D shape measurement - an error analysis. Osten, W.; Kujawinska, M.; Creath, K. (Hrsg.): Proceedings of SPIE. 5144, Optical Measurement Systems for Industrial Inspection III, 2003, S. 372-380

[6] European Machine Vision Association: EMVA Standard 1288 - Standard for Characterization of Image Sensors and Cameras, Release 3.0, November 29, 2010

[7] Bothe, T.: Grundlegende Untersuchung zur Formerfassung mit einem neu-artigen Prinzip der Streifenprojektion und Realisierung in einer kompakten 3D-Kamera, Dissertation, Vollertsen, F., Bergmann, R. (Hrsg.): Strahltechnik, Bd. 32, Bremen, BIAS Verlag, 2008, S. 52-60

[8] Erz, M.: Charakterisierung von Laufzeitkamerasystemen für Lumineszenzlebensdauermessungen, Dissertation, Heidelberg, Universität Heidelberg, 2011, S. 58-62 